\title{
Upregulation of MIAT Regulates LOXL2 Expression by Competitively Binding MiR- 29c in Clear Cell Renal Cell Carcinoma
}

\author{
Yan $\mathrm{Qu}^{\mathrm{a}, \mathrm{c}}$ Haibing Xiao ${ }^{\mathrm{a}, \mathrm{b}}$ Wen Xiao ${ }^{\mathrm{b}}$ Zhiyong Xiong ${ }^{\mathrm{b}}$ Wenjun $\mathrm{Hu}^{\mathrm{a}}$ \\ Yaoying $\mathrm{Gao}^{\mathrm{a}}$ Zeyuan Ru ${ }^{\mathrm{a}}$ Cheng Wang ${ }^{\mathrm{b}}$ Lin Bao ${ }^{\mathrm{b}}$ Kesan Wang ${ }^{\mathrm{b}}$ \\ Hailong Ruan $^{\mathrm{b}} \quad$ Zhengshuai Song $^{\mathrm{b}}$ Ke Chen $^{\mathrm{b}} \quad$ Xiaoping Zhang $^{\mathrm{b}}$ \\ Hongmei Yang ${ }^{a}$
}

aDepartment of Pathogenic Biology, School of Basic Medicine, Tongji Medical College, Huazhong University of Science and Technology, Wuhan, Hubei Province, 'Department of Urology, Union Hospital, Tongji Medical College, Huazhong University of Science and Technology, Wuhan, 'College of Basic Medicine, Xinxiang Medical University, Xinxiang, Henan, China

\section{Key Words}

MIAT $・$ MiR-29c $・$ Loxl2 $\bullet$ ccRCC $・$ CeRNA

\begin{abstract}
Background/Aims: MIAT is a long noncoding RNA (InCRNA) involved in cell proliferation and the development of tumor. However, the exact effects and molecular mechanisms of MIAT in clear cell renal cell carcinoma (ccRCC) progression are still unknown. Methods: We screened the IncRNAs' profile of ccRCC in The Cancer Genome Atlas database, and then examined the expression levels of IncRNA MIAT in 45 paired ccRCC tissue specimens and in cell lines by q-RT-PCR. MTS, colony formation, EdU, and Transwell assays were performed to examine the effect of MIAT on proliferation and metastasis of ccRCC. Western blot and luciferase assays were performed to determine whether MIAT can regulate Loxl2 expression by competitively binding miR-29c in ccRCC. Results: MIAT was up-regulated in ccRCC tissues and cell lines. High MIAT expression correlated with worse clinicopathological features and shorter survival rate. Functional assays showed that knockdown of MIAT inhibited renal cancer cell proliferation and metastasis in vitro and in vivo. Luciferase and western blot assays further confirmed that miR-29c binds with MIAT. Additionally, the correlation of miR-29c with MIAT and Loxl2 was further verified in patients' samples. Conclusion: Our data indicated that MIAT might be an oncogenic IncRNA that promoted proliferation and metastasis of $\mathrm{cCRCC}$, and could be a
\end{abstract} potential therapeutic target in human ccRCC.

Yan Qu and Haibing Xiao contributed equally to this work.

Dr. Xiaoping Zhang

and Dr. Hongmei Yang

KARGER
Department of Pathogenic Biology, School of Basic Med., Tongji Medical College, Huazhong Univ. of Science and Technology, 13 Hangkong Road, Wuhan, 430030, Hubei Prov., (China); Tel. 86-27-85351625, E-Mail xzhang@hust.edu.cn or hyang@hust.edu.cn 
Qu et al.: MIAT Promotes ccRCC by Regulating LOXL2

\section{Introduction}

The incidence of renal cell carcinoma (RCC) has been rising worldwide. It now accounts for nearly 5\% of adult malignancies, with about 63, 990 new cases and 14, 400 deaths estimated in 2017 in the United States alone [1]. Clear cell renal cell carcinoma (ccRCC) is the most common subtype of RCC , accounting for approximately $75-80 \%$ of these tumors [2]. Apart from surgery, it is resistant to both chemotherapy and radiotherapy. Thus, it is important to reveal the molecular mechanisms of ccRCC carcinogenesis to facilitate development of novel cancer biomarkers and appropriate therapeutic strategies.

Long noncoding RNAs (lncRNAs) are a heterogeneous class of transcripts with a minimum length of 200 bases and limited protein-coding potential [3, 4]. Numerous studies have reported that lncRNAs could be crucial players in cancer progression, particularly in terms of dysregulation of gene products that contribute to advanced human tumors [5-7]. Furthermore, lncRNAs could also be considered as diagnostic or prognostic markers due to their clinical association with tumor outcomes [8-10]. More recently, Pandolfi et al. proposed a new regulatory mechanism in which all RNA transcripts harboring miRNA-binding sites can communicate and regulate each other's expression levels by competing specifically for shared miRNAs, thus acting as competing endogenous RNAs (ceRNAs) [11-13].

MIAT, also known as retinal noncoding RNA 2 or Gomafu, was first reported to have been expressed in mitotic progenitors and postmitotic retinal precursor cells [14]. Yan et al. have done excellent work and found that MIAT knockdown clearly ameliorates diabetes mellitus-induced retinal microvascular dysfunction in vivo, and inhibits endothelial cell proliferation, migration, and tube formation in vitro by regulating the expression of the miR150-5p target gene-vascular endothelial growth factor (VEGF) [15]. Zhou et al. found that IncRNA MIAT functions as a ceRNA to up-regulate DAPK2 by sponging miR-22-3p in diabetic cardiomyopathy. MIAT may also play an important role in tumorigenesis. Crea et al. found that MIAT is selectively up-regulated in neuroendocrine prostate cancer and may interact with polycomb genes [16]. A study conducted by Sattari et al. showed that MIAT forms a regulatory loop with OCT4 in malignant mature B cells [17]. However, how MIAT functions in ccRCC pathogenesis remains highly unspecified.

In this study, we found that MIAT has the highest fold change (tumor/normal) in ccRCC among pan-cancer networks by mining the clinical and expression profiles of 14 cancer types (>6000 samples) from The Cancer Genome Atlas (TCGA) Data Portal [18]. Our data also confirmed that MIAT has more prominent expression in renal cancer cell lines and renal cancer tissues. Additionally, our data indicated that MIAT knockdown decreases renal cancer cell proliferation and metastasis in vivo and in vitro. MIAT functions as an oncogene by sponging miR-29c and increasing the expression of Loxl2 in ccRCC. Together, these data contribute to the characterization and the molecular mechanisms of ccRCC progression.

\section{Materials and Methods}

\section{Clinical sample preparation}

A total of 45 paired ccRCC and corresponding noncancerous tissues were obtained sequentially from patients undergoing radical nephrectomy during the period of 2010-2016 in Union Hospital, Wuhan, China. Corresponding noncancerous tissues were acquired at least $5 \mathrm{~cm}$ away from the cancerous site. The study protocol was approved by the ethics committee of Huazhong University of Science and Technology and Union Hospital, and written informed consent was obtained from all participants involved in this study [5].

\section{Cell culture}

Caki-1 cells were cultured in RPMI-1640 supplemented with 10\% fetal bovine serum. ACHN, 786O, and HK-2 cells were maintained in Dulbecco's modified Eagle's medium, supplemented with $10 \%$ fetal bovine serum in a humidified atmosphere of $5 \% \mathrm{CO}_{2}$ maintained at $37^{\circ} \mathrm{C}$ [19].

\section{KARGER}




\section{Cellular Physiology Cell Physiol Biochem 2018;48:1075-1087 \\ \begin{tabular}{l|l} 
DOI: 10.1159/000491974 2018 & $\begin{array}{l}\text { O } 2018 \text { The Author(s). Published by S. Karger AG, Basel } \\
\text { www.karger.com/cpb }\end{array}$
\end{tabular}}

Qu et al.: MIAT Promotes ccRCC by Regulating LOXL2

TCGA analysis

The RNA-seq data of 448 tumors and 67 matched normal samples in ccRCC were downloaded from TCGA Data Portal (http://ibl.mdanderson.org/tanric/_design/basic/query.html) [20].

Oligonucleotide, lentivirus, plasmid, and shRNA

Oligonucleotides corresponding to the target sequences were annealed and cloned into the AgeI and EcoRI sites of the plko.l plasmid (Addgene, Cambridge, USA). Primers were listed as follows: sh-MIAT-1 5'-CCGGGGTGATTACCGTGCACCTTGACTCGAGTCAAGGTGCACGGTAATCACCTTTTTG $-3^{\prime}, \quad 3^{\prime}$-AATTCAAAAAGGTGATTACCGTGCACCTTGACTCGAGTCAAGGTGCACGGTAATCACC-5'; shMIAT-2 5'-CCGGGGTGTTAAGACTTGGTTTCTGCTCGAGCAGAAACCAAGTCTTAACACCTTTTTG-3', 3'-AATTCAAAAAGGTGTTAAGACTTGGTTTCTGCTCGAGCAGAAACCAAGTCTTAACACC-5'. miRNA mimics, inhibitor, and negative(NC)control were purchased from RiboBio (Guangzhou, China).

\section{Quantitative real-time PCR}

All RNA from tissues and cells were extracted with the TRIzol reagent (Invitrogen, Carlsbad, CA) according to the manufacturer's protocol with modifications. cDNAs were synthesized using Rever Ace qPCR RT Kit (TOYOBO, Osaka, Japan). Real-time PCR was performed using SYBR Green Real-Time PCR Master Mix (Roche, Basel, Switzerland) and the ABI ViiA7 qPCR System (Applied Biosystems, Foster, CA). Primers were listed as follows: MIAT 5'- TCTTCATGTCAGAACACGCTTTA-3', 3'-AAGGTCACCCGAGGTCCAA-5'; Loxl2 5'GGAGAGGACATACAATACCAA-3', $3^{\prime}$-GTGACATTCTTCATGGGGT-5'; GAPDH5'-CCTTCATTGACCTCAACTACA-3', 3'-GCTCCTGGAAGATGGTGAT-5'.

\section{Luciferase assays}

Briefly, ACHN and 786-0 cells were seeded in 48- well plates (5000 cells per well) and co-transfected with $100 \mathrm{ng}$ psicheck2 Luciferase vectors containing the $3^{\prime}$ untranslated region of target genes with $50 \mathrm{nM}$ miR-29s mimic or mutant mimic or NC. Forty-eight hours after transfection, a Dual- Luciferase Reporter Assay (Promega, Madison, USA) was performed according to the manufacturer's instructions, as previously described [5].

Colony formation, cell proliferation, EdU, scratch, and cell migration and invasion assays

Colony formation was measured nearly 2 weeks after seeding 1000 cells per well in 6-well plates. Cell proliferation was estimated using the MTS according to the manufacturer's instructions. The EdU assay kit was purchased from RiboBio (Guangzhou,China) and conducted as previously described [19]. Migration and invasion assays were performed using uncoated and Matrigel ${ }^{\mathrm{TM}}$ coated Transwell ${ }^{\circledR}$ inserts according to the manufacturer's instructions. For ACHN migration and invasion, $10^{5}$ and $1.5 \times 10^{5}$ cells were used, whereas 3 $\times 10^{4}$ and $5 \times 10^{4}$ cells were used for 786-0 migration and invasion. All experiments were triplicated.

Xenograft subcutaneously and tail intravenous injection

Tumorigenesis in nude mice was determined as described previously [5]. Mice were injected subcutaneously with the prepared cells at a single site. Tumor onset was measured with calipers at the injection site weekly at different times on the same day. The details of the experimental lung metastasis model were as previously described [5]. All experiments were approved by the Animal Care and Use Committee of Tongji Medical College of Huazhong University of Science and Technology.

\section{Immunohistochemical staining}

Immunohistochemical staining was performed as previously described [21].

\section{Statistical analysis}

All statistical analyses were carried out using SPSS 18.0 statistical software. Continuous data were compared using a Student's two-tailed t-test. Data are presented as mean \pm SEM. In all cases, $p<0.05$ was considered statistically significant. ${ }^{*} \mathrm{p}<0.05 ;{ }^{* *} \mathrm{p}<0.01$. 


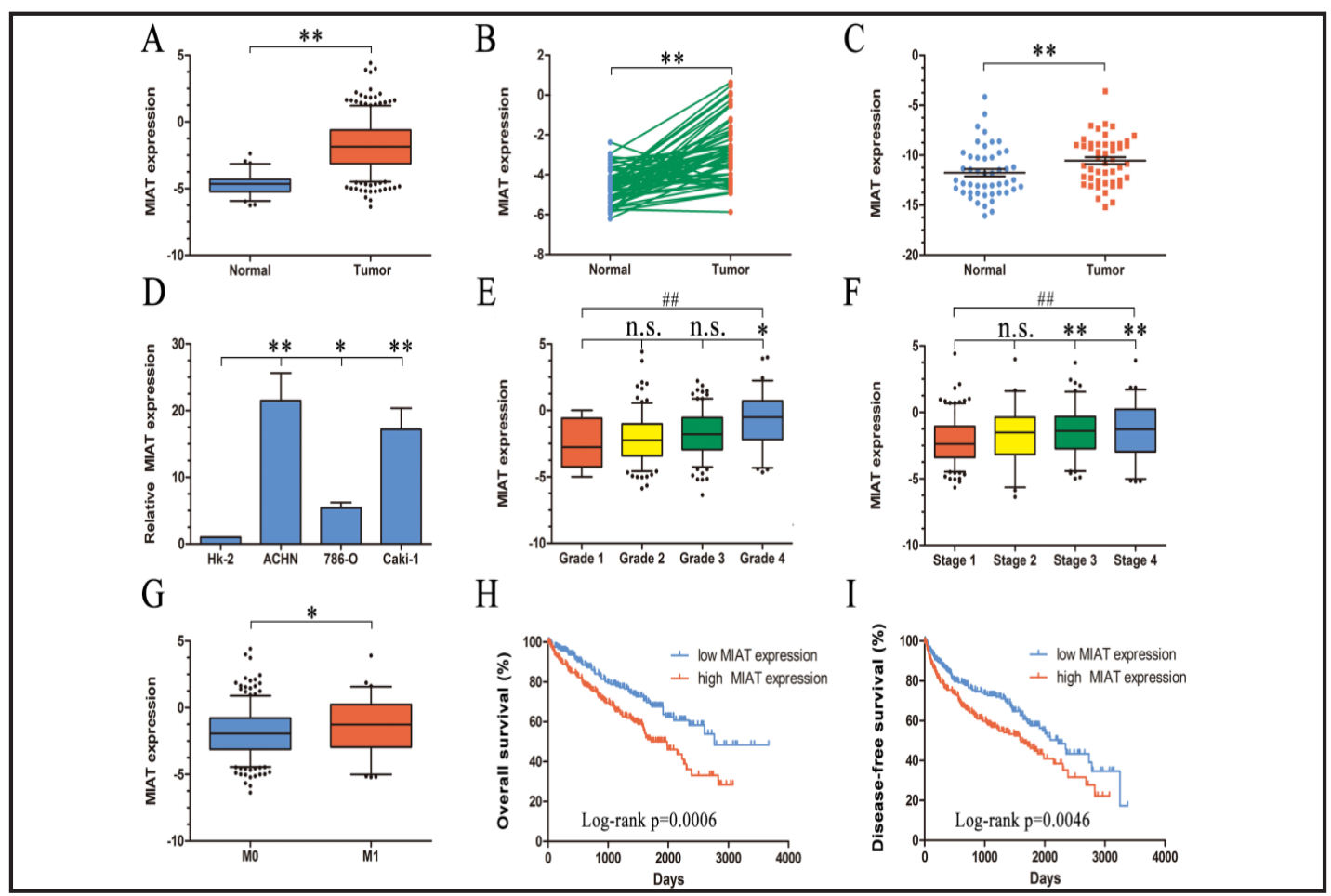

Fig. 1. Expression of MIAT in ccRCC cell lines, tissues and its clinical parameters. (A) MIAT was found to be highly over-expressed in ccRCC tissues compared with normal tissues in the TCGA RNA-seq data $(p<0.001)$. (B) MIAT is significantly increased in primary human ccRCC tissues in comparison to adjacent-normal ccRCC tissues. $\mathrm{n}=55$ for each group. (C) Relative expression of MIAT in 45 pairs of ccRCC tumor tissues and their corresponding adjacent non-cancerous tissues. (D) Real-time PCR analysis of MIAT expression in immortalized human renal tubule epithelial cell line HK-2 and indicated renal carcinoma cell lines. (E, F and G) High MIAT expression was significantly correlated with the TNM grade, stage and metastasis. (H, I) High MIAT expression contributed to significant poorer oval-all survival and disease-free survival in the TCGA database $(\mathrm{n}=480){ }^{*}$, t-test $\mathrm{p}<0.05$; $^{* *}$, t-test $\mathrm{p}<0.01$; \#\#, ANOVA $\mathrm{p}<0.01$.

\section{Results}

MIAT is up-regulated in ccRCC tissues and cell lines and the elevation of MIAT indicates poor prognosis

To determine whether MIAT was involved in the tumorigenesis or development of ccRCC, we first examined the expression of MIAT in TCGA
Table 1. The characteristic of MIAT in clear cell renal cell carcinoma. (MIAT levels were remarkably correlated with grade, TNM stage, metastasis (M) and tumor (T) in ccRCC.)

\begin{tabular}{|c|c|c|c|c|c|}
\hline \multirow[t]{2}{*}{ Characteristic } & & \multirow[t]{2}{*}{ Total $(n=448)$} & \multicolumn{2}{|c|}{ IncRNA-MIAT } & \multirow[t]{2}{*}{ p-Value } \\
\hline & & & Low (224) & High(224) & \\
\hline \multirow[t]{2}{*}{ Gender } & Male & 287 & 144 & 143 & \\
\hline & Female & 161 & 80 & 81 & 0.922 \\
\hline \multirow{2}{*}{ Age } & $<=60$ & 218 & 107 & 111 & \\
\hline & $>60$ & 230 & 117 & 113 & 0.705 \\
\hline \multirow[t]{2}{*}{$\mathrm{T}$} & $\mathrm{T} 1 \& \mathrm{~T} 2$ & 276 & 156 & 120 & \\
\hline & T3\&T4 & 172 & 68 & 104 & 0.001 \\
\hline \multirow[t]{3}{*}{$\mathrm{N}$} & No & 218 & 103 & 115 & \\
\hline & N1 & 16 & 7 & 9 & 0.79 \\
\hline & $\mathrm{Nx}$ & 214 & 114 & 100 & \\
\hline \multirow[t]{2}{*}{ M } & Mo & 377 & 197 & 180 & \\
\hline & M1 & 71 & 27 & 44 & 0.028 \\
\hline \multirow[t]{2}{*}{ Stage } & 1,2 & 260 & 147 & 113 & \\
\hline & 3,4 & 188 & 77 & 111 & 0.001 \\
\hline \multirow{3}{*}{ Grade } & 1,2 & 198 & 115 & 83 & \\
\hline & 3,4 & 245 & 104 & 141 & 0.001 \\
\hline & $\mathrm{x}$ & 5 & 5 & 0 & \\
\hline \multirow{2}{*}{ Recurrence } & No & 349 & 174 & 175 & \\
\hline & Yes & 99 & 50 & 49 & 0.225 \\
\hline
\end{tabular}

Data Portal from TANRIC

(http://ibl.mdanderson.org/tanric/_design/basic/query.html), and found that MIAT had higher expression in ccRCC (tumor vs normal, 448 vs 67) (Fig. 1A). We focused on all paired tissues from TANRIC for further analysis and got the same results (paired tumor vs paired normal, 55 vs 55) (Fig. 1B). To further support this conclusion, we examined the lncRNA MIAT expression by quantitative reverse transcription PCR (q-RT-PCR) and found that MIAT 
Qu et al.: MIAT Promotes ccRCC by Regulating LOXL2

Table 2. Univariate and multivariate analyses of clinicopathological factors for overall survival

\begin{tabular}{lcccccc}
\hline Risk factors & \multicolumn{3}{c}{ Univariate analysis } & \multicolumn{3}{c}{ Multivariate analyses } \\
\hline \multirow{2}{*}{ MIAT expression } & HR & p-Value & $95 \%$ CI & HR & p-Value & $95 \%$ CI \\
Age & 1.069 & 0.013 & $1.014-1.126$ & 1.080 & 0.012 & $1.017-1.147$ \\
Stage & 1.695 & 0.002 & $1.220-2.354$ & 1.564 & 0.008 & $1.124-2.175$ \\
M & 4.308 & 0.000 & $3.046-6.095$ & 2.823 & 0.000 & $1.883-4.233$ \\
Gender & 4.765 & 0.000 & $3.424-6.632$ & 2.468 & 0.000 & $1.683-3.619$ \\
T & 1.022 & 0.896 & $0.737-1.418$ & & & \\
N & 3.597 & 0.000 & $2.588-5.001$ & & & \\
Grade & 2.821 & 0.002 & $1.484-5.365$ & & & \\
Recurrence & 2.421 & 0.000 & $1.692-3.463$ & & & \\
\hline
\end{tabular}

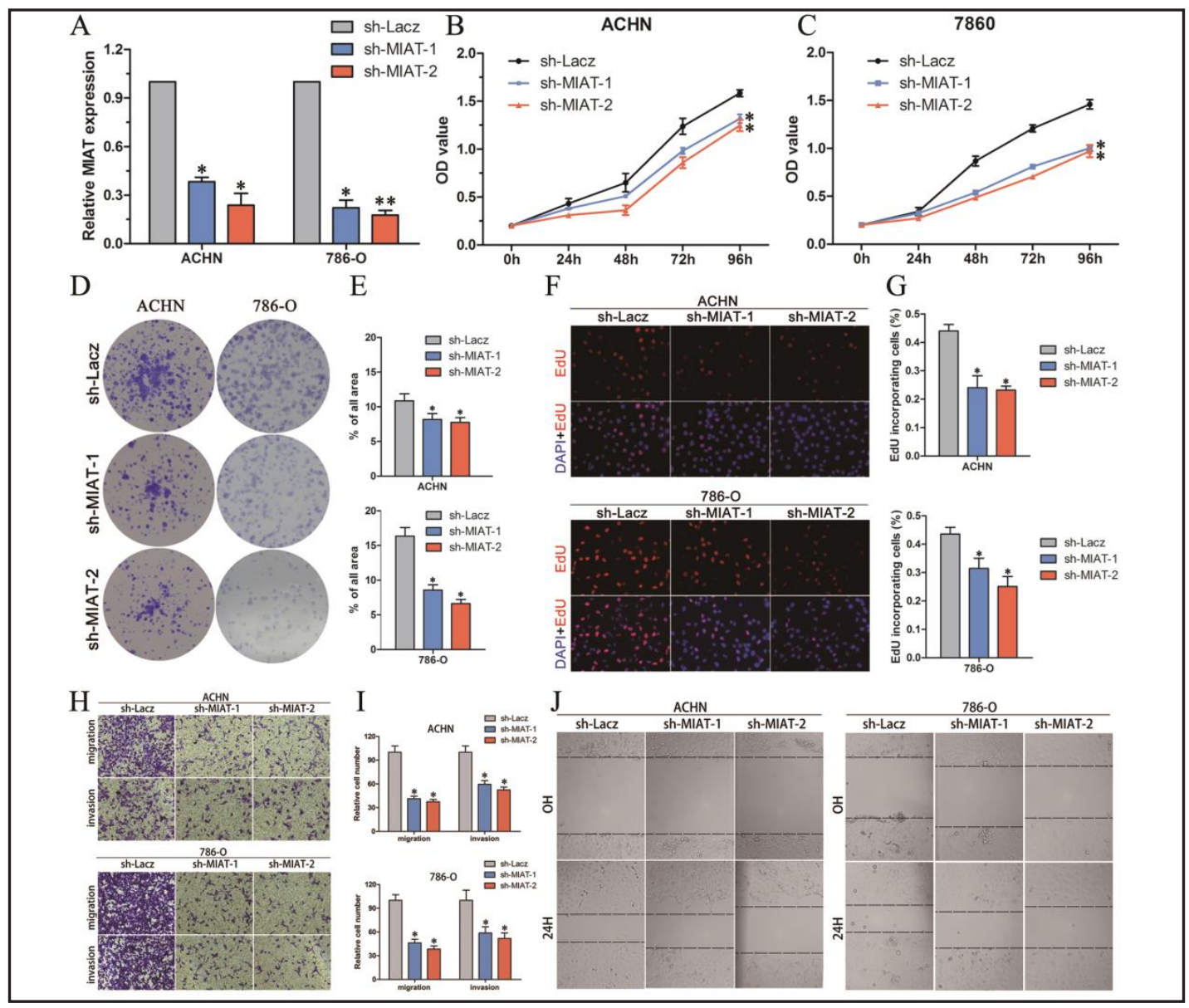

Fig. 2. Knockdown of MIAT inhibited cell proliferation and metastasis in vitro. (A) The efficiency of MIAT silencing in short hairpin RNA-stably transfected renal cancer cell lines ACHN and 786-0. Relative gene ex-

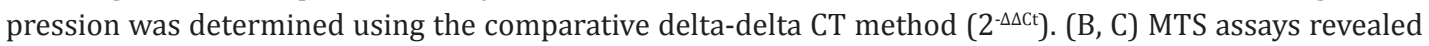
cell growth curves of indicated cells. (D, E) Representative micrographs of crystal violet-stained cell colonies analyzed by clonogenic formation and its relative count. (F, G) EdU assay for renal cancer cells. Representative micrographs (left) and quantification (right) of EdU incorporated-cells in indicated engineered cell lines. Representative photographs were taken at $\times 200$ magnification; number of cells was quantified in ten random images from each treatment group. (H, I) Migration and invasion assay for renal cancer cells. Representative photographs were taken at $\times 200$ magnification; number of migrated cells was quantified in ten random images from each treatment group. (J) Representative micrographs of wound healing assay of the indicated cells. Wound closures were photographed at 0 and 24 hours after wounding. Representative photographs were taken at $\times 100$ magnification. *, t-test $\mathrm{p}<0.05 ;{ }^{* *}$, t-test $\mathrm{p}<0.01$. 
Qu et al.: MIAT Promotes ccRCC by Regulating LOXL2

had higher expression in the 45 renal cancer tissues than their corresponding noncancerous tissues from Union Hospital (Fig. 1C). We obtained similar results from renal cancer cell lines (Fig. 1D).

To evaluate the clinical significance of MIAT, we further analyzed the correlation between MIAT expression and clinicopathological parameters. The results revealed that MIAT levels were remarkably correlated with grade, TNM stage and metastasis in ccRCC (Fig. 1E-1G) (Table 1). Nevertheless, MIAT levels were not associated with other clinical characteristics, including gender $(p=0.922)$, age $(p=0.705)$, and recurrence $(p=0.225)$. Additionally, multivariate Cox regression analysis revealed that high MIAT expression, age, TNM stage, and metastasis were independent predictors of overall survival in ccRCC patients (Table 2). Kaplan-Meier analysis indicated that high MIAT expression was related to poorer overall survival (log-rank test, $\mathrm{p}<0.001$, Fig. $1 \mathrm{H}$ ) and disease-free survival (log-rank test, $\mathrm{p}<0.001$, Fig. 1I). Taken together, these results confirmed that high MIAT expression was related to poor prognosis, and that up-regulated expression of MIAT might be important in ccRCC progression.

\section{Knockdown of MIAT inhibited cell proliferation in vitro}

To investigate the role of MIAT in renal cancer cells, we stably inhibited MIAT in two ccRCC cell lines, ACHN and 786-0, with lenti-viruses carrying shRNA for MIAT and a control nonspecific shRNA (LacZ) (Fig. 2A). Cell proliferation was significantly impaired in renal cancer cells transfected with sh-MIAT, as determined by the MTS (Fig. 2B and 2C) and colony formation (Fig. 2D and 2E) assays. Furthermore, EdU incorporation assays confirmed that sh-MIAT cells (ACHN and 786-0) contained fewer EdU-positive cells with newly synthesized DNA than the control cell populations (Fig. $2 \mathrm{~F}$ and 2G). MIAT also had a mild effect on cell proliferation, migration and invasion in HK-2 cells (data not shown).

Thus, these data suggested that down-regulation of MIAT impaired the proliferation of renal cancer cells.

\section{Knockdown of MIAT inhibited cell migration and Matrigel invasion in vitro}

Next, we explored the effect of MIAT on migration and invasion in ACHN and 786-0 cells. Results showed that silence of MIAT repressed cell motility, migration, and invasion compared with the sh-LacZ group (Fig. 2H and 2I). In detail, knockdown of MIAT suppressed nearly $60 \%$ of the migratory activity of ACHN and 786-O cells, and inhibited $40 \%$ of their invasive activity. In addition, a wound healing assay showed that down-regulation of MIAT impaired the migratory speed of ACHN and 786-O cells compared with the control cells (Fig. 2J). Taken together, these results clearly indicated that knockdown of MIAT expression markedly retarded cell migration and Matrigel invasion in renal cancer in vitro.

\section{miR-29s bind to and suppress MIAT expression}

Recently, many RNA transcripts have been reported to function as ceRNA by competitively binding common miRNAs [22, 23]. It has been reported that MIAT can competitively bind with miR-150 and regulate VEGFA expression in retinal pigment epithelium or with miR155 and regulate DUSP7 in breast cancer $[15,24]$. To further investigate the potential mechanism of MIAT in ccRCC, we used the online starBase, miRcode and DIANA tools to select candidate miRNAs. Three miRNAs (miR-29a, miR-29b, and miR-29c) predicted by all the three tools (Fig. 3A) could bind to the 8593nt-8615nt site of MIAT (Fig. 3B). Then, we constructed a luciferase vector(psi-MIAT) of wild-type MIAT to psicheck2 vector. Using dual-luciferase assays, we found that transfection of psi-MIAT together with miR-29a, miR29b, or miR-29c mimics, but not NC significantly decreased luciferase activities (Fig. 3C). We then graphed the correlation between MIAT and miR-29a, miR-29b-1, miR-29b-2, and miR-29c in TCGA data from TANRIC, and found that only miR-29c had a negative correlation with MIAT (Fig. 3D). We further designed a miR-29c mutant (Fig. 3E) and co-transfected it with psi-MIAT to ACHN and 786-0 cells. Luciferase reporter assays showed that the activity of luciferase linked with psi-MIAT was repressed in a dose-dependent manner in miR-29c 


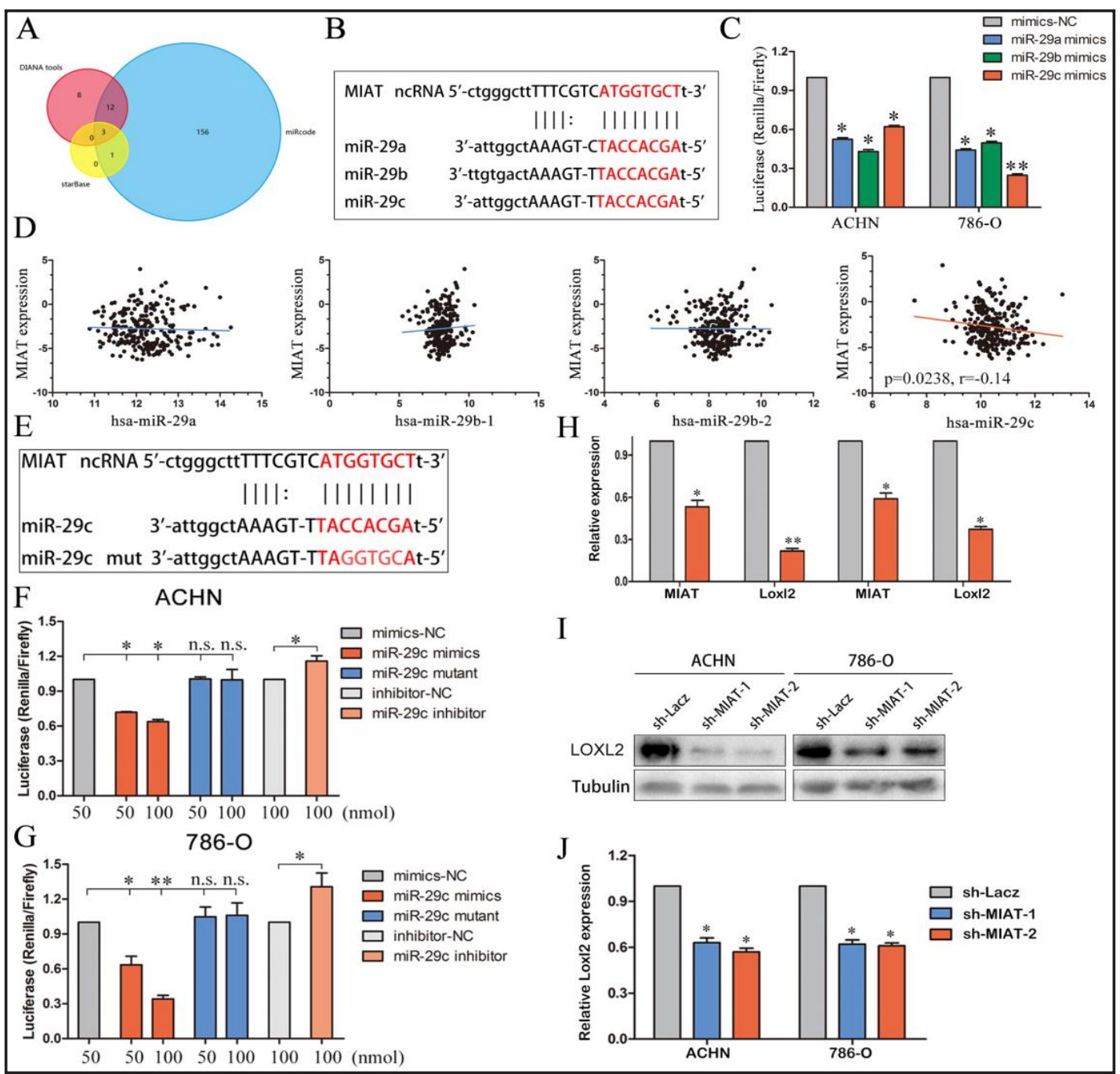

Fig. 3. MiR-29s bind to and suppress MIAT and Loxl2 expression. (A) Online tools including miRcode, starBase and DIANA tools were used to find the candidate upstream miRNAs of MIAT. (B) Schematic miR-29s putative target sites in psi-MIAT. (C) Luciferase reporters harboring putative target sites of psi-MIAT were co-transfected with 50nM of indicated small RNA molecules in ACHN and 786-0 cells. Relative luciferase activity was plotted as the mean \pm SEM of three independent experiments. (D) The correlation of MIAT with miR-29s in ccRCC tissues in TCGA database from TRANIC. (E) The mutant sequence of miR-29c. (F, G) Luciferase assay of psi-MIAT reporters co-transfected with increasing amounts (50 and $100 \mathrm{nmol}$ ) of miR-29c mimic and mutant oligonucleotides in ACHN and 786-0 cell lines, or with $100 \mathrm{nmol}$ miR-29c inhibitor oligonucleotides and negative control of inhibitor. (H) miR-29c decreased the expression of MIAT and Loxl2 by q-RT-PCR. (I) Knockdown MIAT expression decreased the expression of LOXL2 by western blot. (J) Knockdown MIAT expression decreased the expression of Loxl2 by q-RT-PCR.*, t-test $\mathrm{p}<0.05$; **, t-test $\mathrm{p}<0.01$.

mimics transfected ACHN and 786-0 cells, compared with those in NC for mimics or the mutant group. miR-29c inhibitor promoted luciferase activities compared with the control group (Fig. 3F and 3G).

The expression of MIAT was widely decreased in ACHN and 786-O cells after transfection with miR-29c by q-RT-PCR (Fig. 3H). Loxl2, a reported target gene of the miR-29s, was decreased after transfected with miR-29c as well (Fig. 3H) [25]. Further study showed that the decreased expression of MIAT could inhibit Loxl2 expression by q-RT-PCR and western blot (Fig. 3I and 3J). This implied that MIAT sponged miR-29c and released Loxl2 from miR29c. 
Qu et al.: MIAT Promotes ccRCC by Regulating LOXL2

\section{MIAT competed with Loxl2 for miR-29 family binding}

The effects of MIAT expression on endogenous LOXL2 protein were further monitored. The results showed that MIAT knockdown could inhibit the expression of LOXL2, whereas miR-29c inhibitor could relieve the inhibition of LOXL2 by MIAT (Fig. $4 \mathrm{~A}$ and 4B). To further establish a functional connection between miR-29c and MIAT, we tested whether MIAT deregulation was required for regulation of miR-29c on cell proliferation and metastasis. We transfected miR-29c inhibitor or a $\mathrm{NC}$ of the inhibitor to renal cancer cells stably transfected with sh-MIAT or sh-LacZ. We found that the effects of sh-MIAT on proliferation, migration and invasion was partially attenuated by miR-29c inhibitor (Fig. 4C-4H). We conducted additional experiments that after MIAT shRNA suppression, wild-type or a mutant MIAT that failed to bind to miR-29c (all with silent mutations to abolish the effect of MIAT shRNA) or the vector was reintroduced into the cells and then we performed the MTS and migration assays. The wild type MIAT is more powerful than the miR-29c binding-deficient mutant to rescue the phenotypes in renal cancer in vitro and in vivo (Fig. 4I-4K).

Next, we studied the clinicopathological relevance of MIAT, its related molecule miR29c, and Loxl2 expression in 45 matched normal and ccRCC clinical tissue samples. We found significantly elevated expression of Loxl2 and a lower expression of miR-29c in the tumor samples (Fig. 5A and 5B). The TCGA data also showed that high expression of Loxl2 in ccRCC correlated with a poor clinical outcome of ccRCC patients (Fig. 5C). Bivariate correlation analysis showed that expression of MIAT and Loxl2 was significantly correlated with the miR-29c level in ccRCC tissues (Fig. 5D-5F). We obtained the same result in TCGA database (Fig. 5G-5I)

\section{Knockdown of MIAT inhibited cell proliferation and metastasis in vivo}

To further determine whether MIAT affects tumorigenesis in vivo, sh-LacZ/sh-MIAT transfected ACHN cells were inoculated into nude mice. Up to 7 weeks, there was a dramatic 

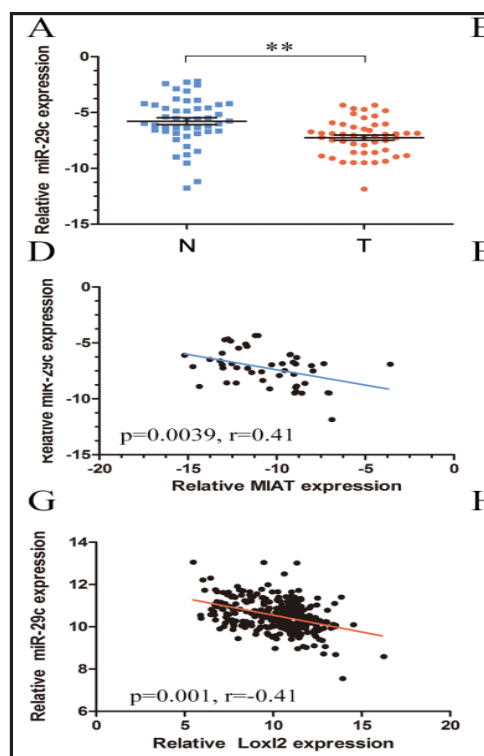
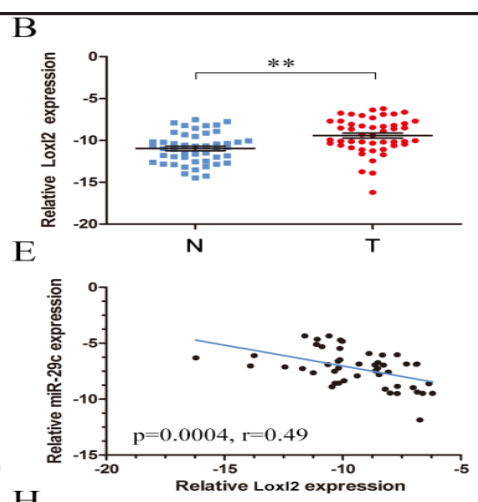

H

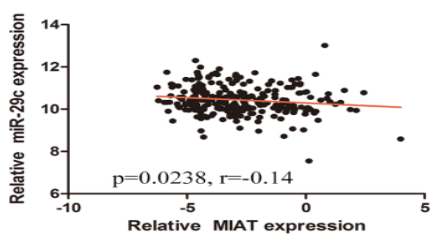

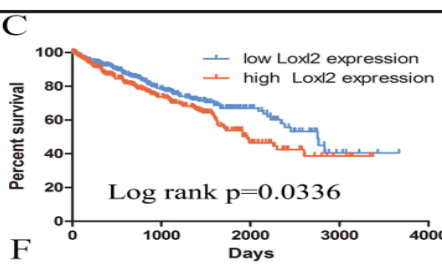

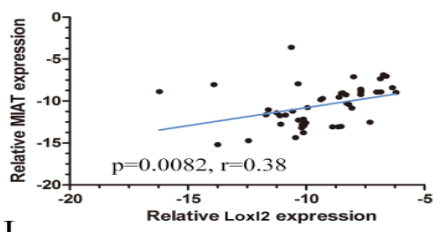

I

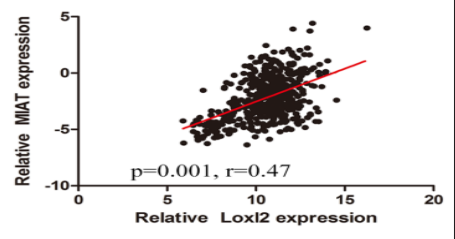

Fig. 5. The correlation between MIAT, Loxl2 and miR-29c. (A, B) Relative expression of Loxl2 and miR-29c in 45 pairs of ccRCC tumor tissues and their corresponding adjacent non-cancerous tissues. (C) High Loxl2 expression contributed to a significant poorer oval-all survival in the TCGA database ( $n=518)$. (D, E and F) The correlation of MIAT, Loxl2 and miR-29c in renal cancer tissue. (G)The correlation between miR-29c and Loxl2. (H)The correlation between miR-29c and MIAT. (I)The correlation between Loxl2 and MIAT. *, t-test $\mathrm{p}<0.05 ; * *$, t-test $\mathrm{p}<0.01$.

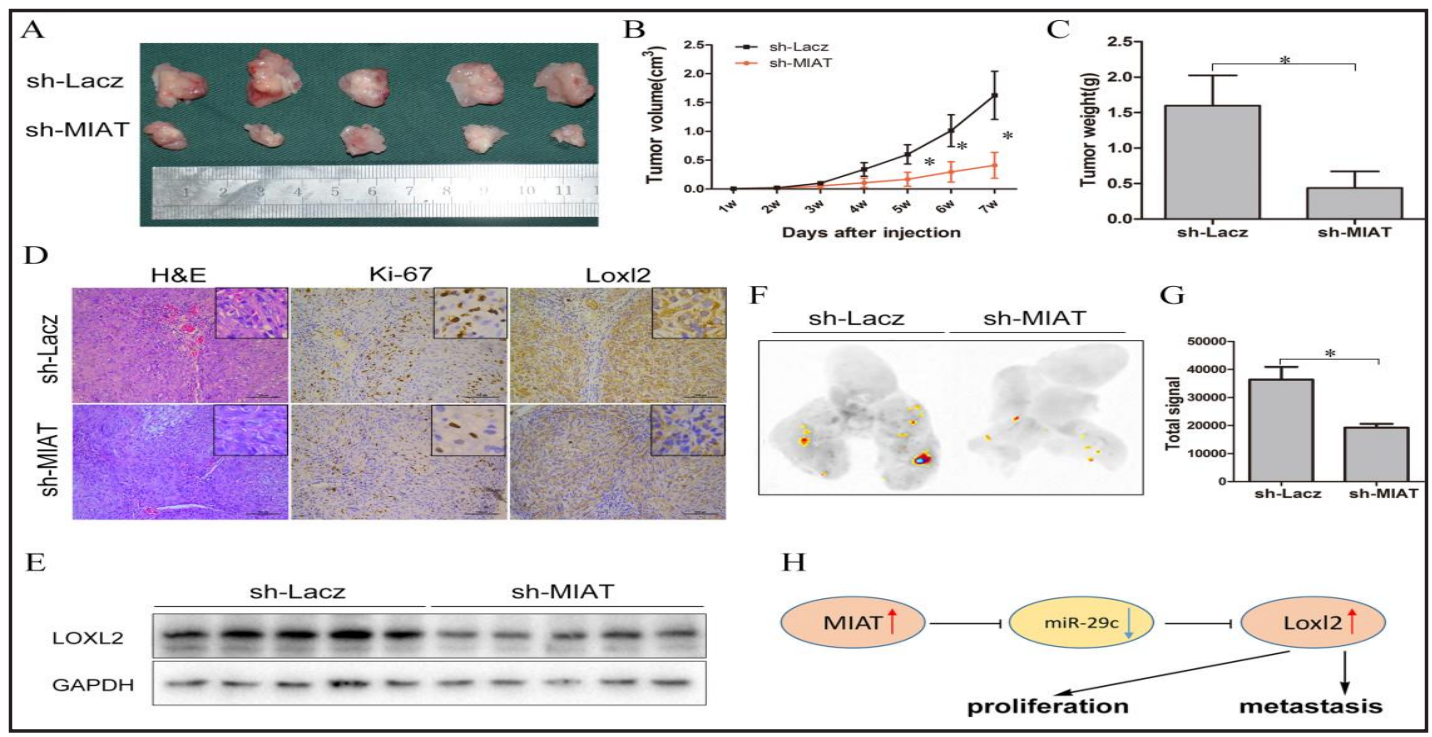

Fig. 6. MIAT induced proliferation in vivo. (A) Photographs of tumors excised 7 weeks after inoculation of stably transfected ACHN cells into the armpit of nude mice ( $n=5)$. (B) Mean tumor volume measured by caliper on the indicated days. (C) Tumor weight of each nude mouse at the end of 7 weeks. (D) H\&E and immunohistochemical staining for Ki-67 and LOXL2 in slices of sectioned implanted tumors formed by indicated cells. Original magnification was $\times 200$. (E) Western blotting confirmed protein expression of LOXL2 in indicated tumors. (F) Representative bioluminescent images of lungs of nude mice after intravenous injection of the indicated renal cancer cell ACHN. (G) Quantifcation analysis of fluorescence signal from captured bioluminescence images. $(\mathrm{H})$ Schematic model of up-regulation of MIAT regulates Loxl2 expression by competitively binding miR-29c in ccRCC *, t-test $\mathrm{p}<0.05$; **, t-test $\mathrm{p}<0.01$. 
Qu et al.: MIAT Promotes ccRCC by Regulating LOXL2

decrease in tumor volume and weight in the sh-MIAT group compared with the sh-LacZ group (Fig. 6A-6C). What's more, we found that the tumors developed from sh-MIAT cells showed less Ki-67 expression than the tumors formed from sh-LacZ cells. Immunohistochemical analysis revealed that tumors developed from sh-MIAT cells showed less LOXL2 expression than the tumors formed in the control (Fig. 6D). In the fresh subcutaneous tumor, we detected the expression of LOXL2 by western blot. There was lower expression of LOXL2 in the shMIAT group than the sh-LacZ group (Fig. 6E). ACHN cells stably expressing sh-MIAT and sh-LacZ were delivered into nude mice by tail vein injection. We found that the total signal of metastatic nodules in the lung was decreased in the sh-MIAT group when compared with the sh-LacZ group (Fig. 6F and 6G).

\section{Discussion}

Recently, many studies have shown that lncRNAs are frequently involved in dysregulation in various tumors and have multiple functions in a wide range of biological processes, such as sustained proliferation, resistance to cell death, activation of invasion, deregulation of cellular energetics, and genomic instability [5, 26-28]. Altered expression of MIAT has been reported to be associated with a susceptibility to myocardial infarction [29]. It has also been proposed that pathways involving this transcript may contribute to the pathophysiology of schizophrenia [30]. However, there are few studies of MIAT in cancer. It has been reported that MIAT is selectively upregulated in neuroendocrine prostate cancer and may interact with polycomb genes [16]. Myc inhibition could increase the expression of MIAT in glioblastoma cells [31]. Luan et al. have found that MIAT promotes breast cancer progression and functions as a ceRNA to regulate DUSP7 expression by sponging miR-155-5p [24]. MIAT expression does not always correlate with tumorigenesis. In the central nervous system, in particular, MIAT is associated with commitment to neuronal differentiation and tumor suppression [31]. However, the function of MIAT in renal cancer has not been reported.

In this study, to determine whether MIAT was involved in the tumorigenesis or development of ccRCC, we first examined the expression of MIAT in the TCGA Data Portal and found that, overall, MIAT exhibited higher expression in tumors than in normal cells in breast cancer, head and neck squamous cell carcinoma, chromophobe renal cell carcinoma clear cell kidney carcinoma, lung adenocarcinoma, and lung squamous cell carcinoma. Further analysis showed that MIAT exhibited higher expression in a group of 45 pairs in ccRCC tumor than in normal tissues, and in renal cancer cell lines. MIAT levels were remarkably correlated with grade, TNM stage, and metastasis in ccRCC. High expression of MIAT was correlated with poor prognosis and shorter survival. Moreover, silencing of MIAT also impaired cell proliferation, migration and invasion in vitro, and inhibited tumorigenesis of ccRCC in vivo.

ceRNAs are endogenous transcripts that, irrespective of their ability to encode for a protein, share common miRNA recognition elements, and hence compete for the binding of normal miRNA molecules. It has become increasingly clear that numerous miRNA-binding sites exist on a wide variety of RNA transcripts, leading to the hypothesis that all RNA transcripts that contain miRNA-binding sites can communicate with and regulate each other by competing specifically for shared miRNAs, thus acting as ceRNAs [22]. Yan et al. showed that MIAT regulates the expression of the miR-150-5p target gene VEGF [15]. Zhou et al. found that lncRNA MIAT functions as a ceRNA to upregulate DAPK2 by sponging miR-22-3p in diabetic cardiomyopathy [32]. However, both studies are not related to tumor research. In fact, the TCGA data showed that both miR-150-5p and miR-22-3p have higher expression in ccRCC, and do not have any negative correlation with MIAT in ccRCC.What's more, the luciferase assay showed that neither miR-150-5p nor miR-22-3p can bind with MIAT in renal cancer cell lines. We also found that the down-regulation of MIAT have little effects on OCT4 expression by q-RT-PCR (data not shown).To elucidate the molecular mechanism of MIAT in tumorigenesis, bioinformatics analysis was performed to explore the potential target gene of MIAT. Only the miR-29s were predicted by all three tools (miRcode, starBase and DIANA tools). 
Qu et al.: MIAT Promotes ccRCC by Regulating LOXL2

The miR-29 family has been observed to be aberrantly expressed in different types of cancer and to be involved in biological functions including cell proliferation, cell cycle, senescence, apoptosis and metastasis [33]. miR-29a, miR-29b, and miR-29c are members of the miR-29 family and act as tumor suppressors in renal cancer tumorigenesis [25]. A luciferase assay showed that, compared with NC, all miR-29s can reduce the luciferase activity of psi-MIAT in the renal cancer cell ACHN and 786-0. The TCGA data from TANRIC showed that there was only a negative correlation between MIAT and miR-29c in renal cancer, so we selected miR-29c for further analysis. A more rigorous luciferase assay showed that, unlike miR-29c mimics, miR-29c mutants cannot reduce the luciferase activity of wildtype MIAT (psi-MIAT).

Loxl2 is a member of the lysyl oxidase gene family, comprising five members, LOX and four LOX-like proteins (LOXL1-4), which can decrease E-cadherin expression by cooperating with the transcription factor Snail [34] or by regulating the methylation status of the E-cadherin gene, thereby promoting epithelial-mesenchymal transition (EMT) [35]. The TCGA data showed that Loxl2 exhibited higher expression in ccRCC and was correlated with poorer prognosis. Nishikawa et al. have reported that miRNA-29s directly regulate LOXL2 expression and inhibit cancer cell migration and invasion in RCC [25]. Our study showed that miR-29c can reduce the expression of MIAT and Loxl2 in the renal cancer cell lines ACHN and 786-0 by q-RT-PCR. We also found that knockdown of MIAT can decrease the expression of LOXL2, whereas miR-29c inhibitors can partly reverse the reduction caused by MIAT knockdown. Knockdown of MIAT represses renal cancer cell proliferation and metastasis can also be partially relieved by miR-29c inhibitor. We also demonstrated that among miR29c, MIAT and Loxl2, there exists a clear correlation in ccRCC.

In summary, it was identified that MIAT could promote ccRCC cell proliferation and metastasis through miR-29c dependent Loxl2 regulation. MIAT may provide a potential therapeutic target for ccRCC.

\section{Abbreviations}

ccRCC (clear cell renal cell carcinoma); MIAT (myocardial infarction associated transcript (non-protein coding)); lncRNA (long noncoding RNA); Loxl2 (lysyl oxidase like 2).

\section{Acknowledgements}

This work was supported by grants from the National Natural Science Foundation of China (No. 81272560, 81773282 to Hongmei Yang and No. 31741032, 81372760, 81672528 to Xiaoping Zhang.), China Postdoctoral Science Foundation (2017M612467).

\section{Disclosure Statement}

No conflict of interests exists.

\section{References}

1 Siegel RL, Miller KD, Jemal A: Cancer Statistics, 2017. CA Cancer J Clin 2017;67:7-30.

-2 Yan BC, Mackinnon AC, Al-Ahmadie HA: Recent developments in the pathology of renal tumors: morphology and molecular characteristics of select entities. Arch Pathol Lab Med 2009;133:1026-1032.

3 Cech TR, Steitz JA: The noncoding RNA revolution-trashing old rules to forge new ones. Cell 2014;157:7794.

4 Guttman M, Russell P, Ingolia NT, Weissman JS, Lander ES: Ribosome profiling provides evidence that large noncoding RNAs do not encode proteins. Cell 2013;154:240-251. 


\section{Cellular Physiology Cell Physiol Biochem 2018;48:1075-1087 \begin{tabular}{l|l} 
DOI: 10.1159/000491974 & $\begin{array}{l}\text { O 2018 The Author(s). Published by S. Karger AG, Basel } \\
\text { www.karger.com/cpb }\end{array}$
\end{tabular}}

Qu et al.: MIAT Promotes ccRCC by Regulating LOXL2

5 Xiao H, Tang K, Liu P, Chen K, Hu J, Zeng J, Xiao W, Yu G, Yao W, Zhou H, Li H, Pan Y, Li A, Ye Z, Wang J, Xu H, Huang Q: LncRNA MALAT1 functions as a competing endogenous RNA to regulate ZEB2 expression by sponging miR-200s in clear cell kidney carcinoma. Oncotarget 2015;10.18632/oncotarget.5357

6 Qu L, Ding J, Chen C, Wu ZJ, Liu B, Gao Y, Chen W, Liu F, Sun W, Li XF, Wang X, Wang Y, Xu ZY, Gao L, Yang Q, Xu B, Li YM, Fang ZY, Xu ZP, Bao Y, Wu DS, Miao X, Sun HY, Sun YH, Wang HY, Wang LH: ExosomeTransmitted lncARSR Promotes Sunitinib Resistance in Renal Cancer by Acting as a Competing Endogenous RNA. Cancer Cell 2016;10.1016/j.ccell.2016.03.004

7 Zhai W, Sun Y, Jiang M, Wang M, Gasiewicz TA, Zheng J, Chang C: Differential regulation of LncRNASARCC suppresses VHL-mutant RCC cell proliferation yet promotes VHL-normal RCC cell proliferation via modulating androgen receptor/HIF-2alpha/C-MYC axis under hypoxia. Oncogene 2016;10.1038/ onc.2016.19

- Wang R, Sun Y, Li L, Niu Y, Lin W, Lin C, Antonarakis ES, Luo J, Yeh S, Chang C: Preclinical Study using Malat1 Small Interfering RNA or Androgen Receptor Splicing Variant 7 Degradation Enhancer ASCJ9(R) to Suppress Enzalutamide-resistant Prostate Cancer Progression. Eur Urol 2017;10.1016/j. eururo.2017.04.005

-9 Zhang S, Zhong G, He W, Yu H, Huang J, Lin T: LncRNA Up-Regulated in Nonmuscle Invasive Bladder Cancer Facilitates Tumor Growth and Acts as a Negative Prognostic Factor of Recurrence. J Urol 2016;10.1016/j. juro.2016.05.107

10 Wu Y, Wang YQ, Weng WW, Zhang QY, Yang XQ, Gan HL, Yang YS, Zhang PP, Sun MH, Xu MD, Wang CF: A serum-circulating long noncoding RNA signature can discriminate between patients with clear cell renal cell carcinoma and healthy controls. Oncogenesis 2016;5:e192.

11 Tay Y, Kats L, Salmena L, Weiss D, Tan SM, Ala U, Karreth F, Poliseno L, Provero P, Di Cunto F, Lieberman J, Rigoutsos I, Pandolfi PP: Coding-independent regulation of the tumor suppressor PTEN by competing endogenous mRNAs. Cell 2011;147:344-357.

12 Salmena L, Poliseno L, Tay Y, Kats L, Pandolfi PP: A ceRNA hypothesis: the Rosetta Stone of a hidden RNA language? Cell 2011;146:353-358.

13 Karreth FA, Reschke M, Ruocco A, Ng C, Chapuy B, Leopold V, Sjoberg M, Keane TM, Verma A, Ala U, Tay Y, Wu D, Seitzer N, Velasco-Herrera Mdel C, Bothmer A, Fung J, Langellotto F, Rodig SJ, Elemento O, Shipp MA, Adams DJ, Chiarle R, Pandolfi PP: The BRAF Pseudogene Functions as a Competitive Endogenous RNA and Induces Lymphoma In vivo. Cell 2015;161:319-332.

14 Ishii N, Ozaki K, Sato H, Mizuno H, Saito S, Takahashi A, Miyamoto Y, Ikegawa S, Kamatani N, Hori M, Saito S, Nakamura Y, Tanaka T: Identification of a novel non-coding RNA, MIAT, that confers risk of myocardial infarction. J Hum Genet 2006;51:1087-1099.

-15 Yan B, Yao J, Liu JY, Li XM, Wang XQ, Li YJ, Tao ZF, Song YC, Chen Q, Jiang Q: IncRNA-MIAT regulates microvascular dysfunction by functioning as a competing endogenous RNA. Circ Res 2015;116:1143-1156.

-16 Crea F, Venalainen E, Ci X, Cheng H, Pikor L, Parolia A, Xue H, Nur Saidy NR, Lin D, Lam W, Collins C, Wang $Y$ : The role of epigenetics and long noncoding RNA MIAT in neuroendocrine prostate cancer. Epigenomics 2016;8:721-731.

17 Sattari A, Siddiqui H, Moshiri F, Ngankeu A, Nakamura T, Kipps TJ, Croce CM: Upregulation of long noncoding RNA MIAT in aggressive form of chronic lymphocytic leukemias. Oncotarget 2016;10.18632/ oncotarget.11099

18 Li JH, Liu S, Zhou H, Qu LH, Yang JH: starBase v2.0: decoding miRNA-ceRNA, miRNA-ncRNA and proteinRNA interaction networks from large-scale CLIP-Seq data. Nucleic Acids Res 2014;42:D92-97.

19 Xiao H, Xiao W, Cao J, Li H, Guan W, Guo X, Chen K, Zheng T, Ye Z, Wang J, Xu H: miR-206 functions as a novel cell cycle regulator and tumor suppressor in clear-cell renal cell carcinoma. Cancer Lett 2016;10.1016/j. canlet.2016.01.032

20 Li J, Han L, Roebuck P, Diao L, Liu L, Yuan Y, Weinstein JN, Liang H: TANRIC: An interactive open platform to explore the function of IncRNAs in cancer. Cancer Res 2015;10.1158/0008-5472.CAN-15-0273

21 Xiao H, Zeng J, Li H, Chen K, Yu G, Hu J, Tang K, Zhou H, Huang Q, Li A, Li Y, Ye Z, Wang J, Xu H: MiR-1 downregulation correlates with poor survival in clear cell renal cell carcinoma where it interferes with cell cycle regulation and metastasis. Oncotarget 2015;6:13201-13215.

22 Tay Y, Rinn J, Pandolfi PP: The multilayered complexity of ceRNA crosstalk and competition. Nature 2014;505:344-352. 


\section{Cellular Physiology Cell Physiol Biochem 2018;48:1075-1087 \begin{tabular}{l|l} 
DOI: 10.1159/000491974 & $\begin{array}{l}\text { O 2018 The Author(s). Published by S. Karger AG, Basel } \\
\text { www.karger.com/cpb }\end{array}$ \\
\cline { 2 - 3 }
\end{tabular}}

Qu et al.: MIAT Promotes ccRCC by Regulating LOXL2

23 Poliseno L, Salmena L, Zhang J, Carver B, Haveman WJ, Pandolfi PP: A coding-independent function of gene and pseudogene mRNAs regulates tumour biology. Nature 2010;465:1033-1038.

24 Luan T, Zhang X, Wang S, Song Y, Zhou S, Lin J, An W, Yuan W, Yang Y, Cai H, Zhang Q, Wang L: Long non-coding RNA MIAT promotes breast cancer progression and functions as ceRNA to regulate DUSP7 expression by sponging miR-155-5p. Oncotarget 2017;10.18632/oncotarget.19190

-25 Nishikawa R, Chiyomaru T, Enokida H, Inoguchi S, Ishihara T, Matsushita R, Goto Y, Fukumoto I, Nakagawa M, Seki N: Tumour-suppressive microRNA-29s directly regulate LOXL2 expression and inhibit cancer cell migration and invasion in renal cell carcinoma. FEBS Lett 2015;589:2136-2145.

-26 Huarte M: The emerging role of IncRNAs in cancer. Nat Med 2015;21:1253-1261.

-27 Posadas EM, McConkey DJ, Chung LWK: Biology and therapy of urological cancer metastasis. Asian Journal of Urology 2016;3:167-169.

28 Yan K, Tian J, Shi W, Xia H, Zhu Y: LncRNA SNHG6 is Associated with Poor Prognosis of Gastric Cancer and Promotes Cell Proliferation and EMT through Epigenetically Silencing p27 and Sponging miR-101-3p. Cell Physiol Biochem 2017;42:999-1012.

29 Vausort M, Wagner DR, Devaux Y: Long noncoding RNAs in patients with acute myocardial infarction. Circ Res 2014;115:668-677.

-30 Rao SQ, Hu HL, Ye N, Shen Y, Xu Q: Genetic variants in long non-coding RNA MIAT contribute to risk of paranoid schizophrenia in a Chinese Han population. Schizophr Res 2015;166:125-130.

-31 Galardi S, Savino M, Scagnoli F, Pellegatta S, Pisati F, Zambelli F, Illi B, Annibali D, Beji S, Orecchini E, Alberelli MA, Apicella C, Fontanella RA, Michienzi A, Finocchiaro G, Farace MG, Pavesi G, Ciafre SA, Nasi S: Resetting cancer stem cell regulatory nodes upon MYC inhibition. EMBO Rep 2016;17:1872-1889.

-32 Zhou X, Zhang W, Jin M, Chen J, Xu W, Kong X: lncRNA MIAT functions as a competing endogenous RNA to upregulate DAPK2 by sponging miR-22-3p in diabetic cardiomyopathy. Cell Death Dis 2017;8:e2929.

-33 Liu L, Bi N, Wu L, Ding X, Men Y, Zhou W, Li L, Zhang W, Shi S, Song Y, Wang L: MicroRNA-29c functions as a tumor suppressor by targeting VEGFA in lung adenocarcinoma. Mol Cancer 2017;16:50.

-34 Peinado H, Del Carmen Iglesias-de la Cruz M, Olmeda D, Csiszar K, Fong KS, Vega S, Nieto MA, Cano A, Portillo F: A molecular role for lysyl oxidase-like 2 enzyme in snail regulation and tumor progression. EMBO J 2005;24:3446-3458.

35 Hase H, Jingushi K, Ueda Y, Kitae K, Egawa H, Ohshio I, Kawakami R, Kashiwagi Y, Tsukada Y, Kobayashi T, Nakata W, Fujita K, Uemura M, Nonomura N, Tsujikawa K: LOXL2 status correlates with tumor stage and regulates integrin levels to promote tumor progression in ccRCC. Mol Cancer Res 2014;12:1807-1817. 